\title{
Complicated pneumonia due to exogenous toxic substances in children
}

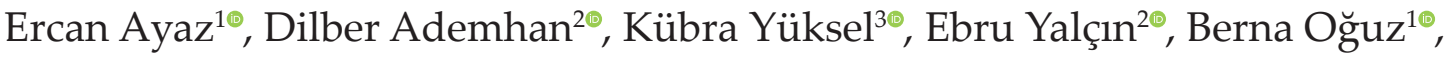 \\ Uğur Özçelik ${ }^{2 \odot}$, Mithat Haliloğlu' ${ }^{1 \odot}$ \\ Departments of ${ }^{1}$ Radiology, ${ }^{2}$ Pediatric Pulmonology and ${ }^{3}$ Pediatrics, Hacettepe University Faculty of Medicine Ankara, Turkey.
}

\begin{abstract}
Background. Toxic substances may be inhaled or aspirated not always by patients themselves but sometimes by healthcare providers unintentionally by nasogastric administrations or medications. Aspirated toxic particles may cause injury in the pulmonary epithelium at various levels of the respiratory tract, leading to a spectrum varying from simple symptoms to severe chronic disease. Imaging features of substances are different depending on their structure and are not well known. Moreover, on follow-up, radiologic findings are not well correlated with clinical findings which progress or recover more rapidly.

Case. In this case series, we report three different chemical pneumonitis due to aspiration of exogenous substances of paint thinner, activated charcoal and liquid paraffin. We present three cases with chest radiographs, distinctive computed tomography findings and follow-up images.

Conclusions. Aspiration of exogenous materials reveals typical imaging findings such as the metallic density of charcoals, fat attenuation of liquid paraffin and necrotic areas within consolidation in hydrocarbon poisoning. Recognition of imaging findings is very important for the diagnosis and characterization of toxic substances.
\end{abstract}

Key words: chemical pneumonia, lipoid pneumonia, activated charcoal aspiration, computed tomography, bronchoscopy.

Chemical pneumonia occurs due to the aspiration of exogenous toxic substances such as volatile hydrocarbons, milk or different kinds of oils that are toxic for the lungs. ${ }^{1}$ Clinical suspicion, detailed information from the family and comprehensive evaluation of the history of the patient are crucial for the diagnosis in order to find out the agent responsible for chemical pneumonia in children. ${ }^{2}$ Like the other forms of aspiration pneumonia, lower lobe infiltrates on chest $x$-ray are important findings of chemical pneumonia along with a sudden onset of dyspnea and coughing. ${ }^{2}$ Iatrogenic reasons, such as prescription of mineral oil or liquid paraffin, administration of activated charcoal

Ercan Ayaz

ercan.ayaz1@gmail.com

Received 19th May 2020, revised 12th June 2020, accepted 18th June 2020. for drug intoxication may also induce chemical pneumonia. ${ }^{3}$ Herein, we present three pediatric patients with aspiration of different exogenous toxic agents and distinctive imaging features along with follow-up and the review of the literature.

\section{Case 1}

A 12-year-old girl with a known diagnosis of mucopolysaccharidosis type 3 was admitted to the emergency department due to ingestion of paint thinner that was noticed by her mother. Gastric lavage was performed immediately, control laboratory findings were normal and she was discharged with a prescription for proton pump inhibitors. Four days later, she presented to the emergency department with fever and malaise. Physical examination revealed bilateral coarse crackles and decreased breath sounds on the left side. Her fever was $39^{\circ} \mathrm{C}$ and her oxygen 
saturation was $80 \%$. There was an infiltration of the left lower lobe and pleural effusion on her chest x-ray (Fig. 1A). The clavicles were short and broad; the ribs were tapered proximally and widened distally consistent with mucopolysaccharidosis. There was also thoracic dextroscoliosis. Her pleural effusion was also confirmed by ultrasound. Sulbactam ampicillin treatment was started with the diagnosis of chemical pneumonitis. However, her clinical findings did not resolve as expected and CT examination was performed. The contrast enhanced CT revealed consolidation of the left lower lobe. Consolidation including multiple nodular hypodense areas were subtle on the parenchymal window of chest CT (Fig. 1B) but more conspicuous on the mediastinal window (Fig. 1C) that did not enhance probably due to necrosis. Pleural effusion was also noted. CT findings were consistent with necrotizing pneumonia due to aspiration of paint thinner. Her treatment was shifted to vancomycin, cefotaxime, and salbutamol, thereafter oxygen saturation normalized, clinical findings resolved and chest x-ray findings regressed in comparison with the initial $x$-ray (Fig. 1D).

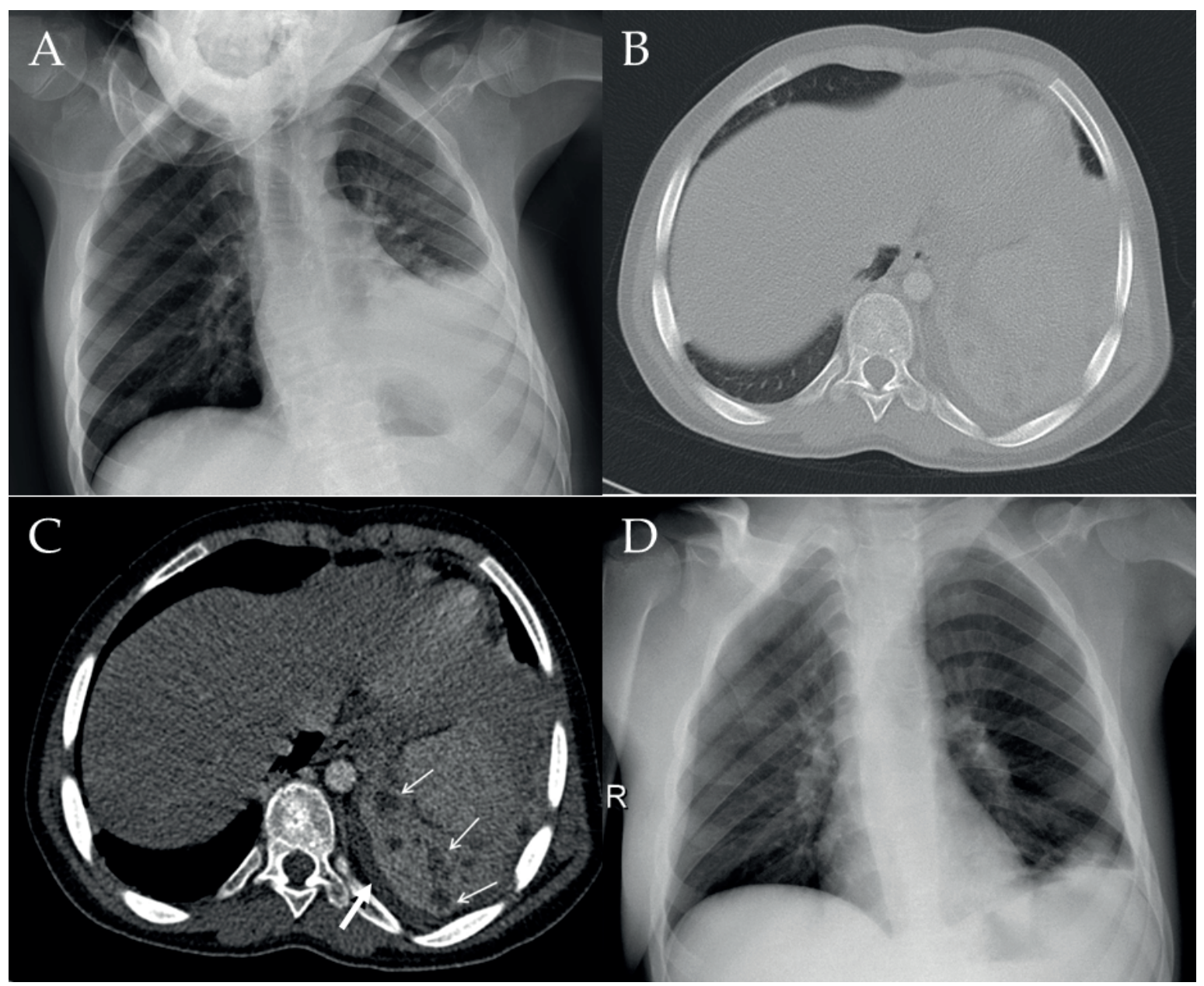

Fig. 1. (A) Chest $x$-ray represents left lower lobe infiltration and left pleural effusion. The clavicles are short and broad. The ribs are tapered proximally and widened distally consistent with mucopolysaccharidosis. There is also thoracic dextroscoliosis. Axial contrast enhanced CT images on parenchymal window (B) and mediastinal window (C) demonstrate left lower lobe consolidation along with left pleural effusion (thick arrow). Hypodense nodular areas within consolidation are more conspicuous (arrows) on mediastinal window. (D) Follow-up chest $\mathrm{x}$-ray 11 days following treatment reveals regression of the effusion and parenchymal findings. 
After a course of two weeks treatment, she was discharged without any complications.

\section{Case 2}

A 3-years and 8-month-old boy was admitted to another institution due to ingested overdose of an unknown drug. Activated charcoal was given by nasogastric tube. The distal tip of the nasogastric tube was placed into the stomach; however, he aspirated a small amount of the activated charcoal due to gastroesophageal reflux. His status deteriorated suddenly and he subsequently required intubation and hospitalized in the intensive care unit for 15 days with the diagnosis of charcoal aspiration. Bronchoalveolar lavage was performed in order to remove the charcoal particles. He was then referred to our pediatric pulmonology clinic for bronchoscopic evaluation. Upon his admission to our hospital, he had a dry cough and his sputum contained charcoal particles. Chest x-ray revealed patchy infiltration of the right lower lobe consistent with charcoal aspiration (Fig. 2A). Chest CT showed multiple tiny nodules forming tree-in-bud appearance at the upper and middle lobes of the right lung and the lingula of the left lung (Fig. 2B). Hyperdense nodules in the right lower lobe revealed significant metallic artifacts due to charcoal

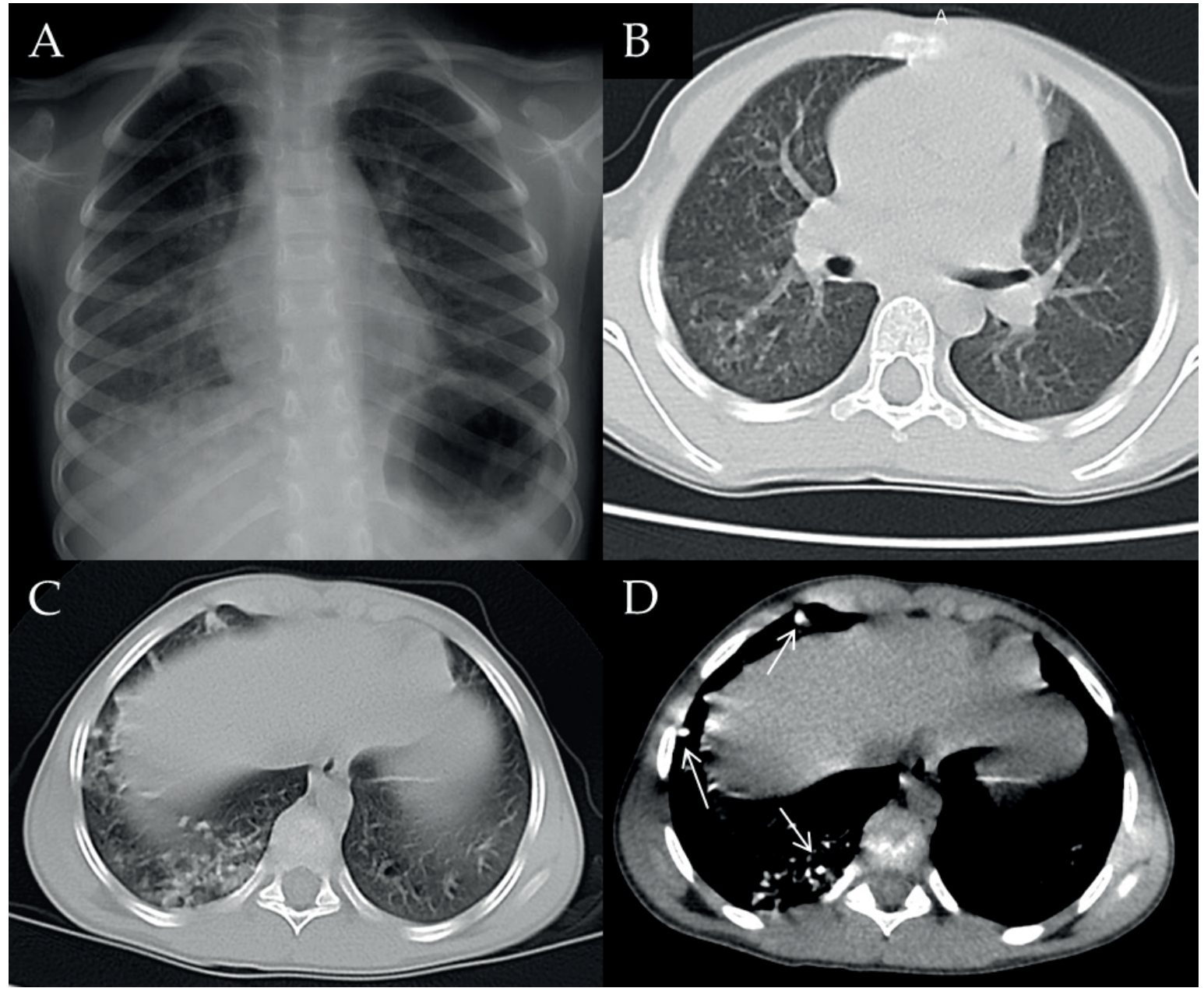

Fig. 2. (A) Chest $x$-ray shows patchy nodular infiltration of the right lower zone. (B) Axial CT with lung parenchymal window shows 'tree in bud' pattern nodular opacities in right upper and middle lobes. Parenchymal (C) and mediastinal (D) window of chest CT at the more caudal section demonstrates metallic densities (arrows) that are consistent with activated charcoal particles at the lower lobe. 
within the nodules that were more prominent on the mediastinal window (Fig. 2C, D). Bronchoalveolar lavage was performed twice at our center to remove remaining charcoal particles with 200 cc saline for each time. After the second lung lavage, charcoal particles were not observed in the sputum anymore. His follow-up chest $\mathrm{x}$-ray performed 3 months later demonstrated nodular opacities at the right lower zone similar to previous imaging with a mild decrease in size and number.

\section{Case 3}

A 7-year-old girl with a history of developmental delay and a chromosomal unstable translocation (46XX der (12) $t(3: 12)$ (q26.3;q24.3)) had a persistent cough for about 3 years and had recurrent lower respiratory tract infections when she presented to the pediatric pulmonology division. She was operated on twice firstly due to anal atresia during the newborn period and secondly due to an inguinal hernia at 5 years of age. She was hospitalized two times for lower respiratory tract infections and used oral antibiotics and inhaled salbutamol for several months. Chest $\mathrm{x}$-ray revealed right middle lobe, right lower lobe, and left lower lobe consolidations (Fig. 3A). She received antibiotic therapy. Chest CT examination revealed that the left lower lobe consolidation and patchy hypodense areas in the consolidation (Fig. 3B,C). There was also patchy consolidation of the right lower lobe.

In the meantime, her mother expressed that for a long time she had been giving her daughter liquid paraffin for chronic constipation. Patients history, excluded infectious and other lung pathologies and CT findings were consistent with lipoid pneumonia due to chronic aspiration of the liquid paraffin. Her bronchoscopy evaluation resulted 70\% lipid-laden macrophage which supported the diagnosis.

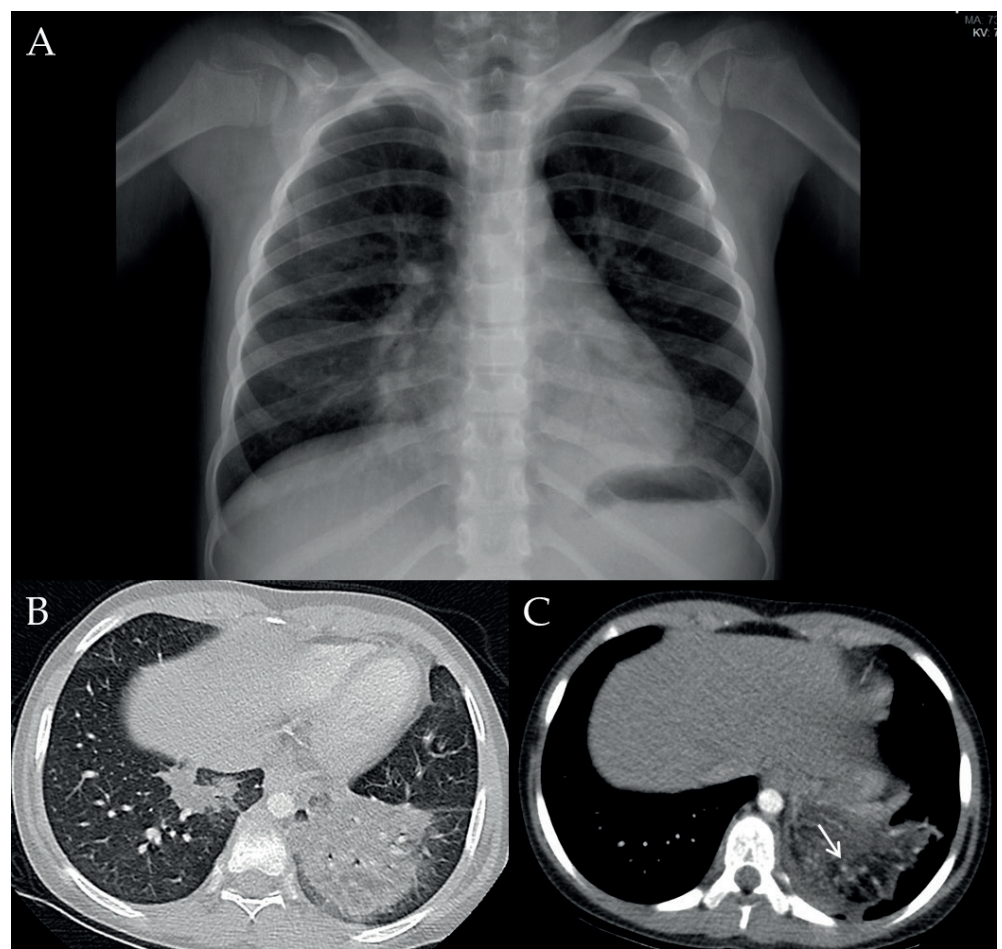

Fig. 3. (A) Chest x-ray demonstrates consolidation of left lower lobe, right lower lobe and right middle lobe. (B) Axial chest CT image on parenchymal window demonstrates right lower lobe and left lower lobe consolidation. (C) On mediastinal window; fat attenuated areas (arrows) within consolidation are more conspicuous consistent with lipoid pneumonia. 
Informed consent and permission were obtained from the parents regarding the publication of these cases.

\section{Discussion}

Paint thinner is a widely available and cheap hydrocarbon product that was commonly abused by homeless children. ${ }^{2}$ On the other hand, careless usage of toxic substances at home, keeping them in the bottle of fruit juice or beverages may facilitate accidental drinking by children. ${ }^{1}$ The most common complication of hydrocarbon intoxication is chemical pneumonia. ${ }^{1}$ However, the prevalence of severe chemical pneumonia after hydrocarbon poisoning is less than $2 \% .^{2}$ Destruction of bronchial and alveolar cells results in interstitial inflammation, intraalveolar hemorrhage and edema, hyperemia, bronchial necrosis, and vascular necrosis. ${ }^{4}$ Imaging features of hydrocarbon aspiration include unilateral or bilateral lung consolidation, well-defined nodules, pneumatoceles (well-defined cavitary nodules), pleural effusion, and spontaneous pneumothorax. ${ }^{1}$ Chemical pneumonia due to inhalation of hydrocarbon products is described as "fire eater's pneumonia" in various articles in the literature. ${ }^{5}$ Apart from pleural effusion and lower lobe consolidation, low attenuating nodular areas, probably consistent with necrosis, is seen in some cases of the adult population similar to our case. ${ }^{5}$ Radiological regression is slower than clinical recovery that may persist up to 8 months. ${ }^{2}$ The main objective of the treatment of hydrocarbon poisoning is to reduce the symptoms. ${ }^{2}$ Medical therapy with antibiotics and steroids related to hydrocarbon poisoning is still controversial. ${ }^{1}$ Since the majority of patients have a benign clinical course, steroids do not seem to offer any additional benefit. ${ }^{6}$ Likewise, in our first patient, we used only antibiotics without steroid treatment. In that patient, control CT was not performed in order to avoid radiation exposure; on her follow-up chest radiographs, there was regression of nodular opacities and pleural effusion.
Activated charcoal is frequently used in pediatric patients with toxic ingestion and the most common complication is vomiting. ${ }^{7}$ Pulmonary aspiration of charcoal is a rare complication with a few case reports in the literature. ${ }^{7} \mathrm{~A}$ majority of the aspiration mechanism is due to aspiration of gastric content including charcoal particles while vomiting, rather than from direct aspiration which may occur by the insertion of the nasogastric tube into the trachea mistakenly. ${ }^{8}$ The incidence of the nasogastric tube placement into the trachea or the distal airways ranges from $0.3 \%$ to $15 \% .{ }^{9}$ Thus, the position of the tube should be verified with an x-ray before the administration of activated charcoal. Graff et al. ${ }^{7}$ reported a case with 'tree in bud nodular opacities in the lower lobes in a patient with direct activated charcoal aspiration. The reason for this appearance is severe bronchiolar impaction with obliteration of the distal bronchioles and contiguous branches by charcoal particles. ${ }^{8}$ In our second case, the same pattern was revealed on the chest $\mathrm{CT}$ in the right lower and middle lobe. Furthermore, chest CT demonstrated metallic artifacts occurred by punctate hyperdense charcoal particles within the nodules. Another complication related to the aspiration of activated charcoal into the airways is pleural involvement. Pleural effusion containing charcoal particles and pneumothorax associated with bronchopleural fistula may be seen if the nasogastric tube is introduced into the distal airways. ${ }^{9}$ Treatment of aspiration is a common problem encountered in pediatric emergency rooms and requires repeated lung lavage and long-term follow-up. ${ }^{7}$

Exogenous lipoid pneumonia occurs in patients with a history of chronic laxative ingestion, and use of oil-based nose drops. The presentation of the clinical scenario could be either acute or chronic. ${ }^{10}$ Acute exogenous lipoid pneumonia is uncommon and a majority of is caused by aspiration of a large quantity of a petroleumbased product or in performers (fire-eaters) who use liquid hydrocarbons for flame blowing. Chronic exogenous lipoid pneumonia is more common and usually results from repeated 
episodes of aspiration or inhalation of oils over an extended period. Although it typically occurs in older patients, it may be seen in children, especially those with a predisposition to aspiration, including intellectual disability and cleft palate. ${ }^{10}$ The severity of the parenchymal disease in lipoid pneumonia depends on the type, amount, frequency, and length of time of aspirated or inhaled oils. Free fatty acids trigger a severe inflammatory reaction that results in focal edema and intraalveolar hemorrhage..$^{10}$ The excessive inflammation can destroy the alveolar walls and the interstitial tissue, and the resultant fibrosis may progress to end-stage lung disease. In the acute phase of lipoid pneumonia, CT may reveal ground glass opacities or consolidation segmental or lobar in distribution and predominantly involves the middle and lower lobes. ${ }^{10}$ Also, CT can demonstrate areas of fat density as low as -30 HU within the consolidation and nodules, a finding diagnostic of lipoid pneumonia like in our third case.

In conclusion, the presented cases emphasize the need for increased awareness among caregivers and pediatricians about the potential hazards of the usage of toxic substances and the importance of comprehensive patient history. Aspiration of exogenous materials may reveal typical imaging findings within consolidations or nodules such as metallic artifact of charcoals, fat attenuation of liquid paraffin and necrotic areas within consolidation in hydrocarbon poisoning. Recognition of radiological signatures is crucial not only for the diagnosis and characterization of the toxic substance but also for management, follow-up and prognosis.

\section{REFERENCES}

1. Bahceci Erdem S, Nacaroglu HT, Isgüder R, Unsal Karkiner CS, Alper H, Can D. Pulmonary complications of chemical pneumonia; a case report. Arch Argent Pediatr 2016; 114: e245-e248.

2. Sen V, Kelekci S, Selimoglu Sen H, et al. An evaluation of cases of pneumonia that occurred secondary to hydrocarbon exposure in children. Eur Rev Med Pharmacol Sci 2013; 17(Suppl 1): 9-12.

3. Salgado IA, Santos CC, Salgado JV, Ferraz PC, Haidar DM, Pereira HA. Exogenous lipoid pneumonia in children: a disease to be reminded of. Rev Assoc Med Bras 2012; 58: 135-137.

4. Lifshitz M, Sofer S, Gorodischer R. Hydrocarbon poisoning in children: a 5-year retrospective study. Wilderness Environ Med 2003; 14: 78-82.

5. Taş S, Durum Y, Karaman C. Fire-eater's pneumonia. Diagn Interv Radiol 2015; 21: 267-268.

6. Makrygianni EA, Palamidou F, Kaditis AG. Respiratory complications following hydrocarbon aspiration in children. Pediatr Pulmonol 2016; 51: 560-569.

7. Graff GR, Stark J, Berkenbosch JW, Holcomb GW 3rd, Garola RE. Chronic lung disease after activated charcoal aspiration. Pediatrics 2002; 109: 959-961.

8. Bairral BQ, Saito M, Morrone N. Activated charcoal bronchial aspiration. J Bras Pneumol 2012; 38: 533534.

9. Nobre LF, Marchiori E, Forte DY, Zanetti G. Pleuropulmonary complications related to pulmonary instillation of activated charcoal. J Bras Pneumol 2013; 39: 396-397.

10. Betancourt SL, Martinez-Jimenez S, Rossi SE, Truong MT, Carrillo J, Erasmus JJ. Lipoid pneumonia: spectrum of clinical and radiologic manifestations. AJR Am J Roentgenol 2010; 194: 103-109. 